\title{
Correction to: Schistosomiasis-from immunopathology to vaccines
}

\section{Donald P. McManus ${ }^{1} \cdot$ Robert Bergquist $^{2} \cdot$ Pengfei Cai $^{1} \cdot$ Shiwanthi Ranasinghe ${ }^{1} \cdot$ Biniam Mathewos Tebeje $^{1}$. Hong You ${ }^{1}$}

Published online: 9 June 2020

(C) Springer-Verlag GmbH Germany, part of Springer Nature 2020

\section{Correction to: Semin Immunopathol} https://doi.org/10.1007/s00281-020-00789-x

The original version of this article inadvertently missed out to display the correct acknowledgement for Fig. 2. The corrected legend of Fig. 2 is given below.

The original article has been corrected.

\section{Donald P. McManus}

Don.McManus@qimrberghofer.edu.au

1 Immunology Department, QIMR Berghofer Medical Research Institute, Brisbane, Queensland, Australia

2 Ingerod, Brastad, Sweden, formerly UNICEF/UNDP/World Bank/WHO Special Programme for Research and Training in Tropical Diseases (TDR), World Health Organization,

Geneva, Switzerland 

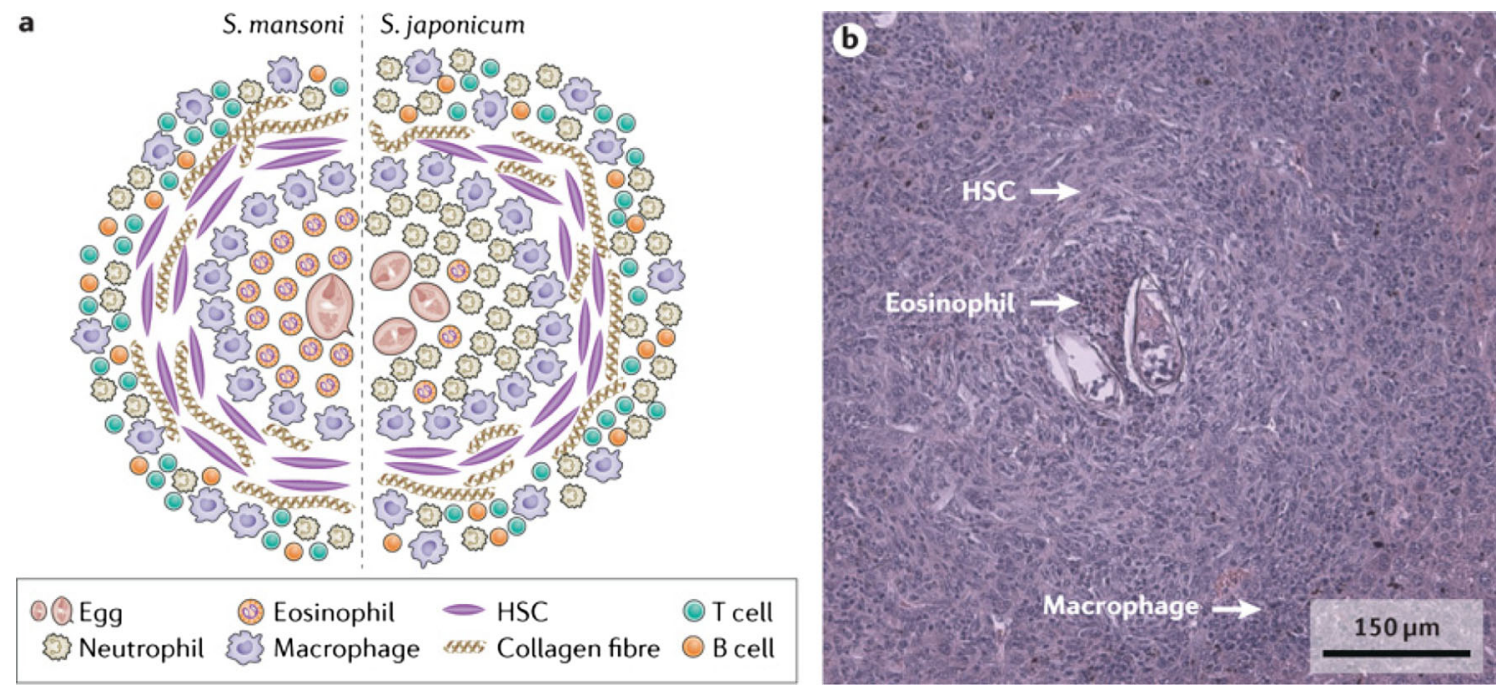

Fig. 2 Features of schistosome-induced granuloma formation. Adult schistosome worm pairs residing in mesenteric veins produce eggs some of which become entrapped in the host's liver (or other organs) tissue where they evoke a dominant CD4(+) $\mathrm{T}_{\mathrm{H}} 2$ immune response mediated by IL- 4 and IL13. This leads to the development of granulomas and fibrosis with hepatic stellate cells, macrophages, lymphocytes, neutrophils, and eosinophils, all identified as major cellular contributors to these events. a Major cellular populations located within and adjacent to the hepatic granuloma induced in either S. japonicum or S. mansoni infection. Whereas a dense population of eosinophils are present at the core of a $S$. mansoni-induced hepatic granuloma, the core in a $S$. japonicum infection is comprised chiefly of neutrophils.

Chemokine-binding proteins secreted by the eggs of $S$. mansoni eggs bind neutrophil chemoattractant C-X-C-motif chemokine ligand 8 (CXCL8), thereby blocking the infiltration of neutrophils to the granuloma. In contrast, these proteins do not bind to eosinophil chemoattractant CC-chemokine ligand 11 (CCL11) and, therefore, do not inhibit the recruitment of eosinophils. b A granuloma in the liver of a $S$. mansoni-infected mouse with hepatic stellate cells (HSCs). Part a adapted with permission from Chuah, C., Jones, M. K., Burke, M. L., McManus, D. P. \& Gobert, G. N. Cellular and chemokine-mediated regulation in schistosome-induced hepatic pathology. Trends Parasitol. 30, 141-150 (2014), Elsevier. Part b courtesy of A. M. O. Kildemoes, University of Copenhagen, Denmark 